\title{
AN OPTIMAL THRESHOLD STRATEGY IN THE TWO-ENVELOPE PROBLEM WITH PARTIAL INFORMATION
}

\author{
MARTIN EGOZCUE, * Universidad de la República de Uruguay \\ LUIS FUENTES GARCÍA, ${ }^{* *}$ Universidade da Coruña
}

\begin{abstract}
In this paper we propose a strategy that gives an optimal lower bound of the average gain for the two-envelope problem within the McDonnell and Abbott (2009) and McDonnell et al. (2011) framework. We obtain this result with partial information about the probability distribution of the envelope's contents.
\end{abstract}

Keywords: Two-envelope problem; threshold strategy; decision theory

2010 Mathematics Subject Classification: Primary 91B06

Secondary 60G40

\section{Introduction}

The two-envelope problem is a well-known paradox in decision theory. It is of interest in diverse fields such as economics, game theory, and stochastic control (see, e.g. Allison and Abbott (2001), Egozcue et al. (2013), Nalebuff (1989), and the references therein).

This problem has many versions (see, e.g. Gardner (1982) and Kraitchik (1930)). In this paper we base our results on the McDonnell and Abbott (2009) and McDonnell et al. (2011) framework, which is described as follows. Consider a one-trial game between a gambling house and one player. A value $x$ is drawn according to a positive random variable $X$. The gambling house places money amounts equal to $x$ and $2 x$ in two identical envelopes. The player chooses one of the envelopes with equal probability, watches the money inside and decides to keep or switch envelopes. The player must find a strategy to maximize his/her payoff.

Brams and Kilgour (1995), Christensen and Utts (1992) and McDonnell et al. (2011), among others, show that when the player has complete information about the distribution of $X$, then a deterministic switching strategy is optimal.

In this paper we obtain a strategy that gives the player a minimum average gain, when he/she knows only the mean $\mu$ and the variance $\sigma^{2}$ of $X$. The paper continues as follows. In Section 2, we present our main results and provide some numerical examples. We finish the paper with concluding remarks.

\section{Main results}

The McDonnell and Abbott (2009) and McDonnell et al. (2011) two-envelope problem framework is the following. Let $Y$ be the random amount of money in the chosen envelope, then

Received 10 July 2013; revision received 11 February 2014.

* Postal address: Department of Economics, Facultad de Ciencias Sociales, Universidad de la República de Uruguay, Montevideo, 11600, Uruguay. Email address: egozcuemj@gmail.com

** Postal address: Departamento de Métodos Matemáticos y de Representacioón, Escuela Técnica Superior de Ingenieros de Caminos, Canales y Puertos, Universidade da Coruña, 15071 A Coruña, Spain. 
the sample space of this stochastic variable is $y \in\{x, 2 x\}$ with $\mathbb{P}(Y=x)=\mathbb{P}(Y=2 x)=\frac{1}{2}$ (conditioned on the smaller amount of the envelope being $X=x$ ). Suppose the player decides whether or not to switch the envelope according to the function $S(y) \in[0,1]$, which is interpreted as the probability of switching after observing $y$. This function can be a simple threshold decision

$$
S_{b}=\mathbf{1}_{[0, b]}(y)= \begin{cases}1 & \text { if } y \in[0, b], \\ 0 & \text { if } y \notin[0, b],\end{cases}
$$

with $b \in R_{+}$. This means that the player switches if $y \leq b$ and does not switch if $y>b$.

The game is repeated many times with different values of $x$ chosen independently from the same distribution. In fact, the average gain compared with never switching, using a strategy $S$, is given by

$$
G_{S}=\frac{1}{2} \mathbb{E}[X(S(X)-S(2 X))],
$$

where $\mathbb{E}[\cdot]$ is the expectation operator (see Equation (2.3) of McDonnell et al. (2011)). Additionally, the average gain using strategy (1) is equal to

$$
G_{S_{b}}=\frac{1}{2} \mathbb{E}\left[X\left(\mathbf{1}_{[0, b]}(X)-\mathbf{1}_{[0, b]}(2 X)\right)\right]=\frac{1}{2} \mathbb{E}\left[X \cdot \mathbf{1}_{(b / 2, b]}(X)\right] .
$$

(see Equation (3.3) of McDonnell et al. (2011)).

In this paper we relax the complete information assumption of $X$, and suppose the player knows only its mean and variance. Our goal is to obtain a strategy that assures a positive average gain with this limited information. In other words, the problem consists of finding the best positive lower bound of

$$
\mathbb{E}\left[X \cdot \mathbf{1}_{\{b / 2<X \leq b\}}\right],
$$

knowing only $\mu$ and $\sigma^{2}$.

The main idea for solving this type of problem is to use an approximation to the discontinuous, piecewise-linear function using a quadratic function (Scarf (1958)).

Theorem 1. Let $X$ be a random variable with mean $\mu$ and variance $\sigma^{2}$. Assume that the player decides according to a threshold strategy as defined in (1).

(a) Then a lower bound of $G_{S_{b}}$ is equal to

$$
\frac{3+2 \sqrt{2}}{b}\left(-m_{2}+\frac{3}{2} b \mu-\frac{1}{2} b^{2}\right)
$$

where $m_{2}=\mu^{2}+\sigma^{2}$.

(b) If, in addition, $\mu^{2} \geq 8 \sigma^{2}$ then the optimal threshold strategy is given by

$$
S_{\sqrt{2 m_{2}}}=\mathbf{1}_{\left[0, \sqrt{2 m_{2}}\right]}(y)= \begin{cases}1 & \text { if } y \in\left[0, \sqrt{2 m_{2}}\right], \\ 0 & \text { if } y \notin\left[0, \sqrt{2 m_{2}}\right],\end{cases}
$$

which assures, at least, an average gain

$$
\underline{G}=(3+2 \sqrt{2})\left(\frac{3}{2} \mu-\sqrt{2 m_{2}}\right) .
$$


Proof. We prove part (a). The idea is to obtain a lower bound of $\mathbb{E}\left[X \cdot \mathbf{1}_{\{b / 2<X \leq b\}}\right]$. Note that we can express

$$
x \cdot \mathbf{1}_{\{b / 2<x \leq b\}}= \begin{cases}x & \text { if } x \in(b / 2, b] \\ 0 & \text { otherwise }\end{cases}
$$

Therefore, we can obtain a lower bound of $x \cdot \mathbf{1}_{\{b / 2<x \leq b\}}$, using a quadratic function, say $Q(x)$, such that

$$
x \cdot \mathbf{1}_{\{b / 2<x \leq b\}} \geq Q(x) \text { for all } x \in\left(\frac{1}{2} b, b\right] .
$$

In Figure 1, we can see the logic behind this argument.

It is easy to see that $b / 2$ and $b$ are the roots of $Q(x)$. Therefore, the function $Q(x)$ is given by

$$
Q(x)=k\left(x-\frac{b}{2}\right)(b-x),
$$

where $k \in R$. Furthermore, as we can see in Figure 1, $Q(x)$ is tangential to $x \cdot \mathbf{1}_{\{b / 2<x \leq b\}}$ at some point in $(b / 2, b)$. Therefore, the function $H(x)=Q(x)-x$ must have a unique double root in $(b / 2, b)$. Thus, we can express

$$
H(x)=-k x^{2}+\left[\frac{3 k b}{2}-1\right] x-\frac{k b^{2}}{2} .
$$

The function (7) has double roots if

$$
\Delta=\left(\frac{3 k b}{2}-1\right)^{2}-4 \frac{k^{2} b^{2}}{2}=0 .
$$

Therefore, we must have $3 k b / 2-1= \pm \sqrt{2} b k$. Consequently, the double root is at $x_{0}=$ $(1 / 2 k)(3 k b / 2-1)$. As we require $x_{0} \in(b / 2, b)$, we discard the negative root and we finally obtain

$$
k=\frac{2}{b(3-2 \sqrt{2})} \text {. }
$$

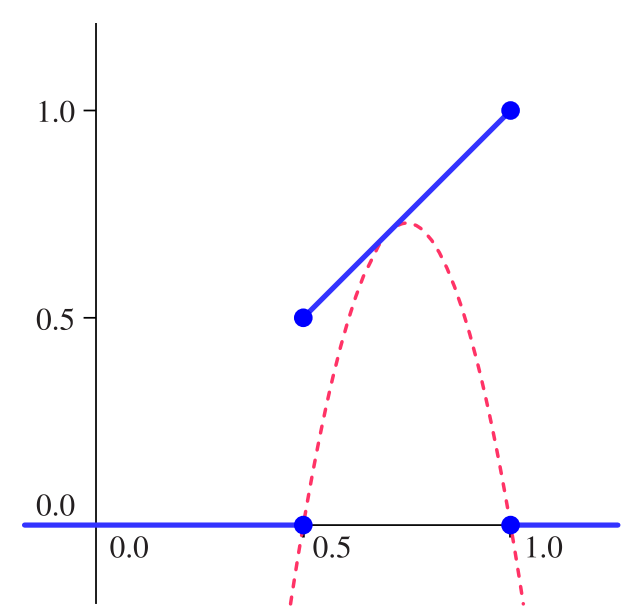

Figure 1: Graph of $x \cdot \mathbf{1}_{\{b / 2<x \leq b\}}$ (bold line) and $Q(x)$ (dashed line), with $b=1$. 
Hence, substituting for $k$ from (8) in (6) we obtain

$$
Q(x)=\frac{2}{b(3-2 \sqrt{2})}\left(-x^{2}+\frac{3}{2} b x-\frac{1}{2} b^{2}\right) .
$$

Finally, substituting for $Q(x)$ from (9) in (5) and taking the expectation on both sides, we obtain

$$
\mathbb{E}\left[X \cdot \mathbf{1}_{\{b / 2<X \leq b\}}\right] \geq \mathbb{E}[Q(X)]=\frac{6+4 \sqrt{2}}{b}\left(-m_{2}+\frac{3}{2} b \mu-\frac{1}{2} b^{2}\right) .
$$

After dividing on both sides by 2 we obtain the desired result.

For part (b), the task is to find the best lower bound $\mathbb{E}\left[X \cdot \mathbf{1}_{\{b / 2<X \leq b\}}\right]$. Therefore, we maximize the right-hand side of (10) with respect to the threshold $b$. Let $f(b)=\left(-m_{2}+\right.$ $\left.3 b \mu / 2-b^{2} / 2\right)(6+4 \sqrt{2}) / b$. It is easy to check that $f(b)$ has a unique maximum when $b=\sqrt{2 m_{2}}$. So, substituting this optimal threshold on the right-hand side of (10), we obtain $(6+4 \sqrt{2})\left(3 \mu / 2-\sqrt{2 m_{2}}\right)$. Hence,

$$
\frac{1}{2} \mathbb{E}\left[X \cdot \mathbf{1}_{\{b / 2<X \leq b\}}\right] \geq \frac{(6+4 \sqrt{2})\left(3 \mu / 2-\sqrt{2 m_{2}}\right)}{2}=(3+2 \sqrt{2})\left(\frac{3 \mu}{2}-\sqrt{2 m_{2}}\right),
$$

which is the desired result. Finally, we can check that this lower bound is nonnegative if $\mu^{2} \geq 8 \sigma^{2}$.

Theorem 1 provides a lower bound of the two-envelope problem when the player knows only the mean and variance of $X$ and uses a threshold strategy. The optimal strategy is simple; the player should switch envelopes if $y \leq \sqrt{2 m_{2}}$, otherwise keep the money in the first envelope.

To gain some insights about the optimal threshold, we demonstrate its relationship with the arithmetic and the geometric means of the envelope's contents. On average, each envelope will contain amounts equal to $\mu$ and $2 \mu$. The arithmetic and geometric means for these amounts are $3 \mu / 2$ and $\sqrt{2} \mu$, respectively. We note that, when $\mu^{2} \geq 8 \sigma^{2}$, the relationship between the optimal threshold, the arithmetic mean and the geometric mean is given by

$$
\sqrt{2} \mu \leq \sqrt{2 m_{2}} \leq \frac{3}{2} \mu .
$$

In summary, strategy (1) enables us to derive a lower bound of the average gain for any distribution function $X$ (as long as $\mu^{2} \geq 8 \sigma^{2}$ holds). In fact, in the next theorem we show that if this condition does not hold, for any threshold strategy, there always exists a random variable $X$ such that (2) is 0 .

Theorem 2. Let $\mu, \sigma \in R_{+}$and $b>0$. Then there exists a positive random variable $X$ with mean $\mu$ and variance $\sigma^{2}$, with $\mu^{2}<8 \sigma^{2}$, such that

$$
G_{S_{b}}=0 .
$$

Proof. The idea is to find, for every $b$, a discrete distribution function $X=\left\{x_{1}, x_{2}\right\}$ such that

$$
0<x_{1}<\frac{1}{2} b<b<x_{2} \text {. }
$$

As we can see, for this distribution we have $G_{S_{b}}=0$. Let $\mathbb{P}\left(X=x_{1}\right)=p, \mathbb{P}\left(X=x_{2}\right)=1-p$ and

$$
x_{1}=\mu-t \sigma, \quad x_{2}=\mu+t^{-1} \sigma,
$$


for $t=\sqrt{(1-p) / p} \in(0, \infty)$. We can check that $\mathbb{E}[X]=\mu$ and $\boldsymbol{V}[X]=\sigma^{2}$. We will show that for every $b$ we can find $t$ such that (11) holds. The condition $0<x_{1}<b / 2$ together with $t>0$ is equivalent to

$$
\max \left\{\frac{\mu-b / 2}{\sigma}, 0\right\}<t<\frac{\mu}{\sigma} .
$$

Whereas, the inequality $x_{2}>b$ is equivalent to

$$
\frac{1}{t}>\frac{b-\mu}{\sigma} .
$$

We will divide the study of conditions (12) and (13) in the two possible cases.

(1) If $\mu \geq b$, it is easy to see that both conditions (12) and (13) hold. To find the random variable $X$, we simply set $t \in((\mu-b / 2) / \sigma, \mu / \sigma)$ and the required distribution function follows.

(2) If $\mu<b$, condition (13) can be rewritten as $t<\sigma /(b-\mu)$. Hence, conditions (12) and (13) can be written as

$$
\max \left\{\frac{\mu-b / 2}{\sigma}, 0\right\}<t<\min \left\{\frac{\mu}{\sigma}, \frac{\sigma}{b-\mu}\right\} .
$$

To prove that there exists a $t$ such that (14) holds, we need to check whether the following inequality holds:

$$
\max \left\{\frac{\mu-b / 2}{\sigma}, 0\right\}<\min \left\{\frac{\mu}{\sigma}, \frac{\sigma}{b-\mu}\right\} .
$$

We need to consider two cases.

(a) If $\mu \leq b / 2$ then (15) trivially holds.

(b) Finally, if $b / 2<\mu<b$, since $(\mu-b / 2) / \sigma>0$, then $\max \{(\mu-b / 2) / \sigma, 0\}=$ $(\mu-b / 2) / \sigma$. Thus, we must consider two cases.

(i) If $\min \{\mu / \sigma, \sigma /(b-\mu)\}=\mu / \sigma$ the condition (15) trivially holds.

(ii) Whereas, if $\min \{\mu / \sigma, \sigma /(b-\mu)\}=\sigma /(b-\mu)$ then we need to check that

$$
\frac{\mu-b / 2}{\sigma}<\frac{\sigma}{b-\mu},
$$

which is equivalent to proving that $\sigma^{2}>(\mu-b / 2)(b-\mu)$. Let $h(b)=$ $(\mu-b / 2)(b-\mu)$. It is easy to see that $h(b)$ achieves a unique maximum at $b=3 \mu / 2$, with $h(3 \mu / 2)=\mu^{2} / 8$. By hypothesis $\mu^{2}<8 \sigma^{2}$, hence, we have

$$
h(b)=\left(\mu-\frac{b}{2}\right)(b-\mu) \leq \frac{\mu^{2}}{8}<\sigma^{2} .
$$

This completes the proof of this theorem. 


\subsection{An illustration}

We now present numerical simulations of our results. The description of the simulation is as follows. Assume that the player knows only the mean and the variance of $X$. According to Theorem 1, the player uses strategy (3). We now use Monte Carlo simulations in order to estimate the player's average gain, assuming that the money is drawn from two different probability distributions (which, obviously, are not known by the player).

1. The random variable $X$ has a uniform distribution on $[A, B]$, with density function equal to

$$
f(x)=\mathbf{1}_{[A, B]}(x),
$$

with $A=8-\sqrt{3}$ and $B=8+\sqrt{3}$. Thus, $\mu=8$ and $\sigma^{2}=1$.

2. The random variable $X$ has a gamma density function with scale $\theta>0$ and shape $k>0$ parameters given by

$$
f(x)=\frac{1}{\theta^{k} \Gamma(k)} x^{k-1} \mathrm{e}^{-x / \theta} \mathbf{1}_{(0, \infty)}(x),
$$

with $\theta=\frac{1}{8}$ and $k=64$. Hence, $\mu=k \theta=8$ and $\sigma^{2}=k \theta^{2}=1$.

Since $m_{2}=65$, the minimum average gain a player would expect, using (3), is equal to 3.486.

As we see in Figure 2, equation (2) for the uniform and gamma distributions are 4 and 3.977, respectively.

Remark 1. We remark that Theorem 1 finds a lower bound $\underline{G}$ when the player knows only the mean and variance of $X$. Therefore, as we can see from Figure 2, the average gain for these specific random variables $X$, do not coincide with $\underline{G}$. As we can expect, the average gain for both distributions are well above this value.

\section{Concluding remarks}

In this paper we obtained an optimal threshold strategy that provides a lower bound of the average gain within the McDonnell and Abbott (2009) and McDonnell et al. (2011) framework.

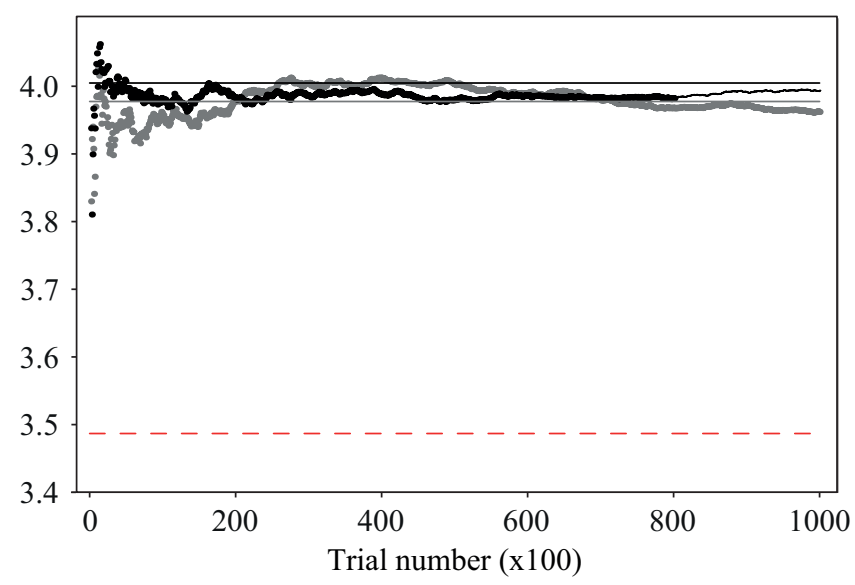

FIGURE 2: Empirical average gain (equation (2)) for 100000 independent trials, for a uniform distribution (black line) and for a Gamma distribution (grey line). The average gain for the uniform and gamma distributions (black and grey straight lines, respectively) and equation (4) (dashed line). 
We do this assuming that there is limited information about the probability distribution of the envelope's contents. Indeed, we require only the mean and the variance of $X$. This paper could be extended in several directions. First, we have restricted the analysis to a threshold strategy. A future task is to consider other strategies, such as Cover's proposal (see McDonnell and Abbott (2009)). Second, analyzing to see if these new strategies improve the lower bound (4) also remains for future work.

\section{Acknowledgements}

We are grateful to a reviewer for providing us with a number of comments and suggestions that have significantly improved the readability of the paper. We acknowledge research support by our respective universities as well as by the Agencia Nacional de Investigación e Innovación (ANII) and FEDER funds.

\section{References}

Allison, A. And Aвbott, D. (2001). Control systems with stochastic feedback. Chaos 11, 715-724.

Brams, S. J. AND Kilgour, D. M. (1995). The box problem: to switch or not to switch. Math. Magazine 68, 27-34.

Christensen, R. And UtTs, J. (1992). Bayesian resolution of the 'exchange paradox'. Amer. Statistician 46, $274-276$.

Egozcue, M., Fuentes García L. And Zitikis, R. (2013). An optimal strategy for maximizing the expected real-estate selling price: accept or reject an offer? J. Statist. Theory Practice 7, 596-609.

Gardner, M. (1982). Aha! Gotcha: Paradoxes to Puzzle and Delight. W. H. Freeman, San Francisco, CA.

KraitchiK, M. (1930). Le paradoxe des cravates. In La Mathematique des Jeux, Imprimerie Stevens Frères, Bruxelles. McDonnell, M. D. And Аввотt, D. (2009). Randomized switching in the two-envelope problem. Proc. R. Soc. London A 465, 3309-3322.

MCDonnell, M. D. et al. (2011). Gain from the two-envelope problem via information asymmetry: on the suboptimality of randomized switching. Proc. R. Soc. London A 467, 2825-2851.

Nalebuff, B. (1989). Puzzles: the other person's envelope is always greener. J. Econom. Perspectives 3, 171-181.

SCARF H. (1958). A min-max solution of an inventory problem. In Studies in the Mathematical Theory of Inventory and Production, Stanford University Press, pp. 201-209. 